\title{
USO DAS TECNOLOGIAS E O ISOLAMENTO SOCIAL: UMA REVISÃO
} SISTEMÁTICA

Vitor Hugo Sales Ferreira; Universidade de Brasília (UnB);vitorhugosalesferreira@gmail.com

Luiza Rosa Bezerra Leão; Universidade de Brasília (UnB); luizarosaleao@gmail.com

Andrea Mathes Faustino; Universidade de Brasília (UnB); andreamathes@unb.br

\section{RESUMO}

Introdução: As tecnologias, por serem um suporte às demandas das perdas funcionais do envelhecimento, aos aspectos psicológicos e sociais visam melhorar a qualidade de vida dos idosos, principalmente aqueles em situação de isolamento social. Objetivo: Identificar as tecnologias de apoio a pessoas idosas em isolamento social. Método: Trata-se de revisão integrativa, cuja a pergunta norteadora foi: "Quais as tecnologias de apoio a pessoas idosas em situação de isolamento social, mais utilizadas no Brasil e no Mundo?” A buscas foram realizadas a partir de publicações científicas indexadas nas bases de dados: SCOPUS, MEDLINE via PubMed, LILACS e SCIELO. O período de análise das publicações foi entre os anos de 2014 a 2019, nos idiomas inglês, português e espanhol. Descritores utilizados: idoso, tecnologia”, isolamento social. Ao final das buscas, 33 publicações atenderam aos critérios de elegibilidade e compuseram o estudo. Resultados: Diante dos achados extraídos dos estudos selecionados, percebeu-se que houve predominância de uso de tecnologias da informação para se lidar com o isolamento social entre pessoas idosas, em especial, de redes sociais ou de realidades aumentada, além das vídeo-chamadas. Muitos estudos se pautavam em tecnologias duras das áreas de comunicação e informação. Houve uma predominância de publicações realizadas pelos Estados Unidos $(n=12)$, seguido da Austrália $(n=5)$. Apenas um artigo havia sido desenvolvido no Brasil. Conclusão: As alternativas para minimizar o fenômeno do isolamento social entre idosos, são ainda muito recentes, sendo as tecnologias mais utilizadas como suporte a idosos em isolamento social aquelas associadas as tecnologias de comunicação.

Palavras-chave: Idoso; Tecnologia; Isolamento Social.

Agradecimentos: à Coordenação de Aperfeiçoamento de Pessoal de Nível Superior (CAPES) pela concessão de bolsa de mestrado à Vitor Hugo Sales Ferreira e Luiza Rosa Bezerra Leão. 